\title{
Cluster of differentiation 44- and octamer-binding transcription factor-4-positive stem-like osteosarcoma cells involved in tumor development
}

\author{
LAN-MEI LIU ${ }^{1}$, HONG-AI SUN ${ }^{2}$, XIAO LI ${ }^{1}$, YANG CHEN ${ }^{1}$, BAO-FU WEI ${ }^{3}$ and XIU-JU LI ${ }^{4}$ \\ ${ }^{1}$ Department of Orthopedics, Linyi People's Hospital, Linyi, Shangdong 276000; \\ ${ }^{2}$ Red Cross Blood Center of Linyi City, Linyi, Shangdong 276001; ${ }^{3}$ Department of Orthopedics, \\ Shandong Provincial Hospital, Jinan, Shandong 250021; ${ }^{4}$ Department of General Surgery, \\ Linyi Cancer Hospital, Linyi, Shangdong 276000, P.R. China
}

Received June 2, 2014; Accepted January 22, 2015

DOI: $10.3892 / 01.2015 .3163$

\begin{abstract}
Osteosarcoma (OS) is an aggressive primary bone cancer that usually affects children and young adolescents. Previous studies have demonstrated the implications of a small sub-population of cancer stem cells on treatment failure and tumor recurrence. The present study analyzed the characteristic features of the stem-like cells within the human OS-55 cell line. It was identified that $2.3 \%$ of the OS-55 cells were cancer stem-like side population (SP) cells. Following treatment with verapamil, the population of SP cells was reduced to $0.7 \%$. The sphere formation assay revealed that the OS cells were able to rapidly form tumor spheres (also known as sarcospheres). Immunofluorescence analysis identified that the OS-55 cells expressed the cluster of differentiation 44 , octamer-binding transcription factor-3/4A and Nanog stem cell surface markers. The results of the present study suggest that, as with other tumors, OS also contains a sub-population of cancer stem-like cells, which may have important implications in cancer diagnosis and treatment.
\end{abstract}

\section{Introduction}

Osteosarcoma (OS) is the eighth most common malignancy in children and young adolescents, and accounts for $2.4 \%$ of all pediatric malignancies. Following diagnosis, the mean survival rate of patients with OS is less than five years $(1,2)$. In total, $\sim 20 \%$ of patients are at the metastatic lung stage at the time of diagnosis. Unsuccessful treatment strategies lead to mortality. Therefore, an understanding of the molecular mechanisms that underlie tumorigenesis is important.

Correspondence to: Dr Xiu-Ju Li, Department of General Surgery, Linyi Cancer Hospital, 6 Linyuandong, Lanshan, Linyi, Shandong 276000, P.R. China

E-mail: lixiuju85496@gmail.com

Key words: cancer stem cells, metastasis, tumor recurrence, drug resistance, adenosine triphosphate-binding cassette transporters
Previous studies concerning a number of different cancers have identified the existence of a small sub-population of cancer-initiating cells known as cancer stem cells (CSCs), which exhibit stem cell-like properties and are responsible for tumor metastasis, invasion and chemotherapy resistance (3-8). The existence of CSCs has also been demonstrated in human OS, which possesses characteristic features of stem cells and expresses the octamer-binding transcription factor (Oct)3/4A and Nanog stem cell surface markers $(9,10)$. A noteworthy feature of these cells is their ability to efflux Hoechst 3342 dye and other chemotherapeutic drugs (10), which ultimately results in chemoresistance and tumor recurrence.

CSCs can be isolated based upon the expression of stem cell surface markers, such as cluster of differentiation (CD) 44 and CD133 (11), or by the Hoechst 33342 dye exclusion technique by fluorescence-activated cell sorting (FACS) (12). With FACS, the cells that exclude Hoechst 33342 dye are named the side population (SP) cells. Another technique is the sphere formation assay, in which individual cancer cells form a spherical colony in a defined serum-free medium (10). In each of these methods, the isolated cancer stem-like cells exhibit resistance to chemotherapy and apoptosis, a high level of self-renewal and a higher level of stem cell surface markers. However, the molecular and signaling pathways involved in these functions are yet to be elucidated. The present study attempted to isolate and characterize the stem-like cells from the OS-55 cell line. The isolated cancer stem-like cells were further analyzed for the expression of the stem cell surface markers, Oct-3/4A, CD44 and Nanog.

\section{Materials and methods}

Cell lines and culture conditions. Samples of stage III aggressive primary human OS with lung metastasis (OS-55 cells) were obtained from the American Type Culture Collection (Manassas, VA, USA). Subsequent to isolation, the samples were washed in phosphate-buffered saline (PBS) containing antibiotics, and then incubated overnight in Dulbecco's modified Eagle's medium (DMEM)/F12 (Gibco Life Technologies, Carlsbad, CA, USA) containing $500 \mathrm{U} / \mathrm{ml}$ penicillin, $500 \mu \mathrm{g} / \mathrm{ml}$ streptomycin and $1.25 \mu \mathrm{g} / \mathrm{ml}$ amphotericin B (Gibco 
A

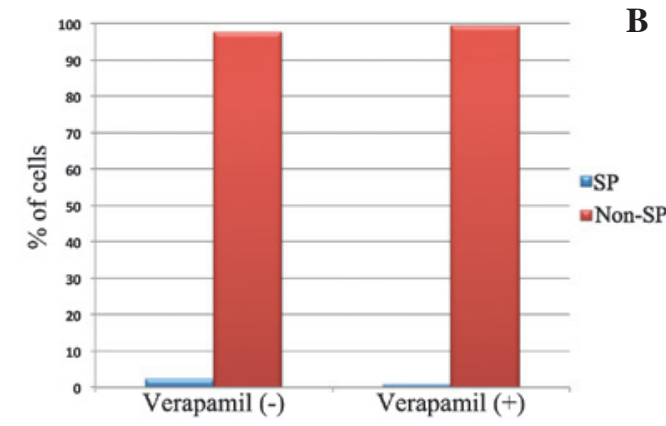

C

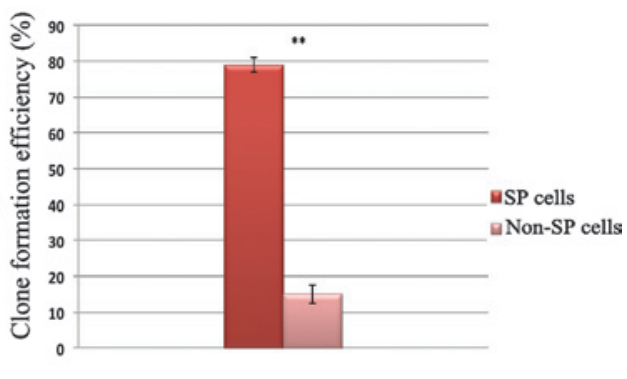

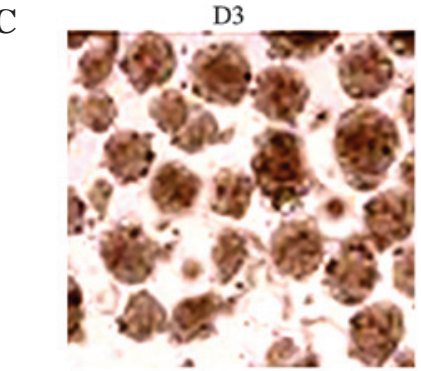

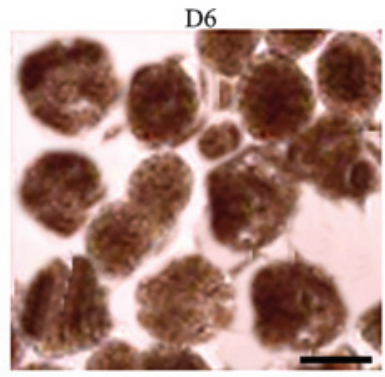

Figure 1. (A) Quantification graph of dot plot fluorescence-activated cell sorting analysis revealing that $2.3 \%$ of the human osteosarcoma OS-55 cells were cancer stem-like side population (SP) cells. Following treatment with verapamil, the population of SP cells was reduced to 0.7\%. (B) The sphere formation assay revealed that the sorted SP cells generated significantly more sarcospheres compared with the non-SP cells. The bar represents the standard deviation. ${ }^{* *} \mathrm{P}<0.01$ vs. non-SP cells. (C) Representative phase contrast images of monoclonal spheres formed by the OS-55 SP cells. The sarcospheres were generated on day three, and increased in size over time. Scale bar, $25 \mu \mathrm{m}$.

Life Technologies). Enzymatic digestion was performed using $1.5 \mathrm{mg} / \mathrm{ml}$ collagenase (Gibco Life Technologies) and $20 \mu \mathrm{g} / \mathrm{ml}$ hyaluronidase in PBS for $1 \mathrm{~h}$. Next, the cells were cultured in DMEM with $10 \%$ fetal bovine serum (Sigma-Aldrich, St. Louis, MO, USA) supplemented with antibiotics, and then maintained in T-75 flasks at $37^{\circ} \mathrm{C}$ in a humidified $5 \% \mathrm{CO}_{2}$ and 95\% air atmosphere. The cells that reached $90 \%$ confluency were removed from the culture flask using Trypsin-EDTA (0.25\% Trypsin/53 mM EDTA; Sigma-Aldrich), washed and then suspended in $10 \%$ DMEM. The cell count was performed using a hemocytometer.

FACS analysis. The experimental groups were as follows: i) A control group consisting of cells treated with Hoechst 33342 dye $(n=7)$; and ii) a drug-treated group consisting of cells treated with verapamil (G.D. Searle LLC Division of Pfizer Inc., Chicago, IL, USA) and Hoechst 33342 dye (n=7). In total, $\sim 10^{6}$ cells $/ \mathrm{ml}$, which had been incubated with $10 \%$ DMEM, were labeled with Hoechst 33342 stock containing $5 \mu 1 / \mathrm{ml}$ bis-benzimide (Sigma-Aldrich), alone or in combination with $0.8 \mu \mathrm{l} / \mathrm{ml}$ verapamil. Next, the cells were resuspended in $500 \mu \mathrm{l} \mathrm{Hank}$ 's balanced salt solution containing $10 \mathrm{mM}$ HEPES (Sigma-Aldrich) for FACS analysis. Finally, the cells were counterstained with $2 \mu \mathrm{g} / \mathrm{ml}$ propidium iodide (Sigma-Aldrich).

Sarcosphere formation assay. A sphere formation assay was performed as previously described (10). Firstly, the cells were plated at a density of 60,000 cells/well in ultra-low attachment six-well plates containing serum-free DMEM/F12 medium supplemented with $\mathrm{N}-2,10 \mathrm{ng} / \mathrm{ml}$ epidermal growth factor and $10 \mathrm{ng} / \mathrm{ml}$ human basic fibroblast growth factor. The culture was then analyzed for sphere formation each day until day seven. Images were captured using an inverted phase contrast microscope (Eclipse TS100; Nikon Corporation, Tokyo, Japan). Subsequent to seven days of culturing, the total number of spheres (also known as sarcospheres) were generated using FACS. The sorted SP and non-SP cells were then quantified.

Immunofluorescence staining. The sorted OS-55 SP and non-SP cells were fixed in Cytofix ${ }^{\mathrm{TM}}$ solution (BD Biosciences, Franklin Lakes, NJ, USA) and incubated for $20 \mathrm{~min}$ at $4^{\circ} \mathrm{C}$. Subsequent to blocking in donkey serum (Sigma-Aldrich) for $20 \mathrm{~min}$, the cells were incubated with a goat anti-Oct3/4A antibody (dilution, 1:200; cat. no. sc-8628; Santa Cruz Biotechnology, Inc., Dallas, TX, USA) overnight at $4^{\circ} \mathrm{C}$. Next, the cells were incubated with a rhodamine red-conjugated donkey anti-goat antibody (dilution, 1:200; Jackson ImmunoResearch Laboratories, Inc., West Grove, PA, USA). For the CD44 and Nanog immunofluorescence analysis, the cells were stained with a fluorescein isothiocyanate-conjugated anti-human Nanog or CD44 antibody (dilution, 1:5; eBioscience, Inc., San Diego, CA, USA). Human embryonic stem cells were used as a positive control. Finally, the cells were observed under a confocal fluorescence microscope (LSM 150; Zeiss, Oberkochen, Germany), and images were analyzed and processed using Adobe Photoshop CS4 (Adobe Systems, Inc., San Jose, CA, USA).

Statistical analysis. A one-way analysis of variance and Student's t-test were performed in order to identify any significant differences between the treatment and control groups. $\mathrm{P}<0.01$ was considered to indicate a statistically significant difference. 

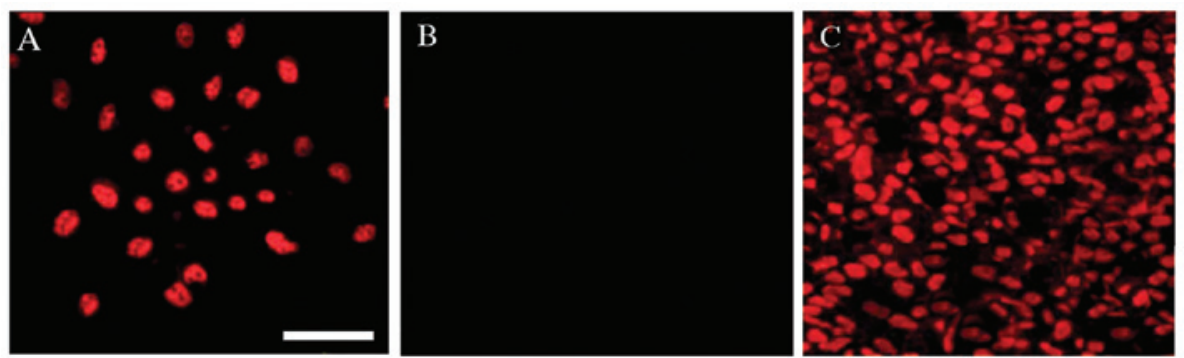

Figure 2. Representative immunofluorescence staining images revealing the expression of octamer-binding transcription factor (Oct)3/4A protein in the human osteosarcoma OS-55 cell line. (A) Sorted SP cells positive for Oct3/4A expression. (B) Non-SP cells demonstrating null expression. (C) Positive control human embryonic stem cells. Scale bar, $50 \mu \mathrm{m}$.
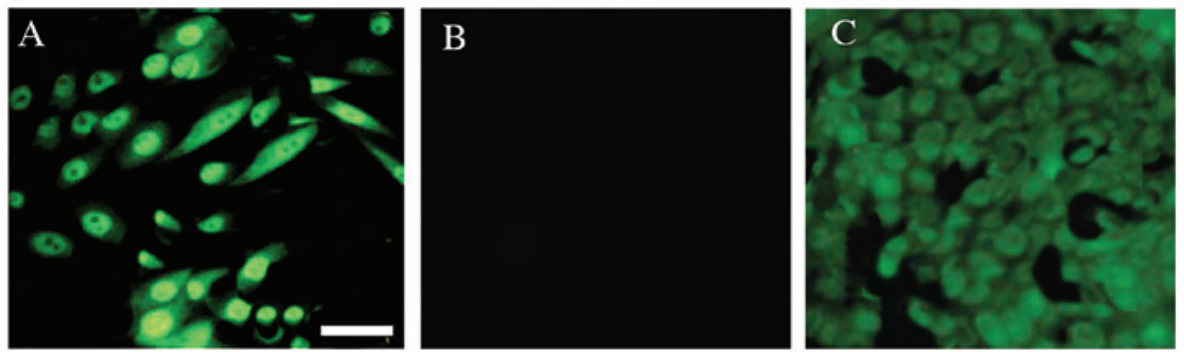

Figure 3. Representative immunofluorescence staining images revealing the expression of Nanog protein in the human osteosarcoma OS-55 cell line. (A) Sorted SP cells positive for Nanog expression. (B) Non-SP cells demonstrating null expression. (C) Positive control human embryonic stem cells. Scale bar, $50 \mu \mathrm{m}$.
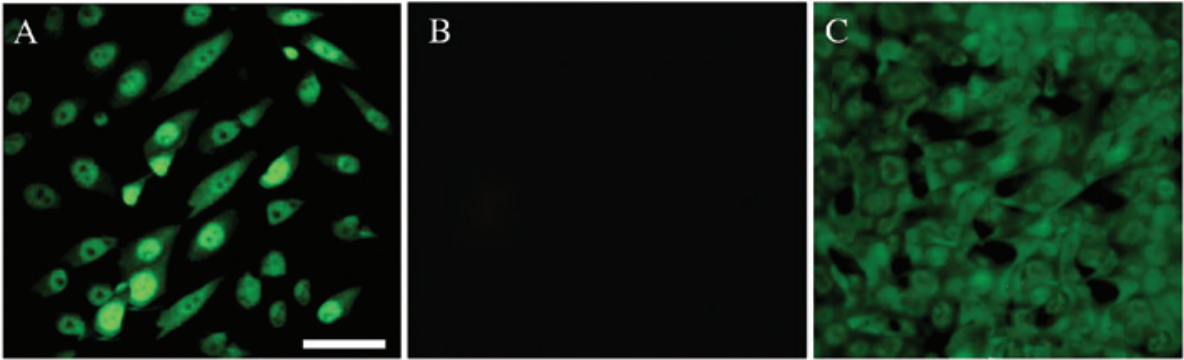

Figure 4. Representative immunofluorescence staining images revealing the expression of cluster of differentiation (CD) 44 protein in the human osteosarcoma OS-55 cell line. (A) Sorted SP cells positive for CD44 expression. (B) Non-SP cells demonstrating null expression. (C) Positive control human embryonic stem cells. Scale bar, $50 \mu \mathrm{m}$.

\section{Results}

The present study analyzed the human OS-55 cell line using Hoechst 33342 dye exclusion by FACS in order to identify and characterize the small stem-like population of cells. As shown in Fig. 1A, it was identified that $2.3 \%$ of the OS-55 cells were cancer stem-like SP cells. The percentage of SP cells decreased to $\sim 0.7 \%$ following treatment with verapamil, which blocks the action of adenosine triphosphate-binding cassette $(\mathrm{ABC})$ transporters. This confirms the property of drug exclusion by the cancer stem cells, which, as with other solid tumors, express ABC transporter proteins. The sorted OS-55 SP and non-SP cells were also examined in order to investigate their sarcosphere-forming ability. The total number of sarcospheres formed by the SP cells was significantly higher than that of the non-SP cells (Fig. 1B). SP cells rapidly formed spheres on day three, which increased in size over time (Fig. 1C). In addition, the sorted SP and non-SP cells were subjected to immunocytochemical analysis in order to examine the expression of stem cell surface markers. Using confocal microscopy, the bright nuclear staining of Oct3/4A (Fig. 2) and Nanog (Fig. 3) was detected in the SP cells. Furthermore, cytoplasmic CD44 (Fig. 4), which was evident in the cancer stem-like SP cells, also demonstrated high expression in the SP cells. However, very little or null expression of the stem cell surface markers was observed in the non-SP cells. Therefore, these data suggested that the human OS-55 cell line contains a small population of cancer stem-like cells, which demonstrate high levels of self-renewal and drug resistance.

\section{Discussion}

A number of previous studies have identified the presence of a small population of tumor-initiating cells known as CSCs, which are able to initiate rapid tumor proliferation $(3,13,14)$. These cells possess stem cell-like features and are responsible 
for chemotherapy resistance and tumor recurrence following conventional treatment strategies (8). In addition, the overexpression of ABC transporters, such as ABCG2, in CSCs actively contributes to multi-drug resistance and results in treatment failure. Therefore, it is essential to identify the characteristic features of CSCs in order to provide effective treatment strategies. In the present study, it was revealed that $2.3 \%$ of the human OS-55 cell population were cancer stem-like SP cells. This decreased to $0.7 \%$ following treatment with verapamil, which confirmed an overexpression of $\mathrm{ABC}$ transporters in the SP cells. The sphere formation assay is able to determine the self-renewal capacity of CSCs in several types of tumor (5). The results of the present study demonstrated that the sorted SP cells rapidly formed sarcospheres on day three, and that the size of the spheres increased with time. Furthermore, the cancer stem-like SP cells generated a larger number of sarcospheres than the non-SP cells. In accordance with these findings, a previous study demonstrated that OS OS99-1 and MG63 cells were able to form sarcospheres (15).

Oct3/4A, CD44 and Nanog are markers associated with stem cell-like properties, including self-renewal, pluripotency, tumorigenesis and tumor invasion $(14,16,17)$. An overexpression of these factors appears to increase the self-renewal capacity of cells. The significance of these proteins has been well documented in a number of human cancers $(18,19)$. A previous study reported the elevated expression of Oct3/4A, Oct3/4B and Nanog mRNA in the human OS99-1 cell line compared with three other cell lines that were analyzed (15). The positive expression of Oct3/4A, Nanong and CD44 can be used as a specific marker for the diagnosis of cancer metastasis (20). It was also identified that Oct3/4A and Nanog are highly expressed in OS CSCs (15) and are involved in tumor metastasis. In accordance with these findings, the present study demonstrated that human OS-55 cells have enhanced Oct3/4A and Nanog expression in the nuclei of the cancer stem-like SP cells compared with the non-SP cells. CD44 is a member of a family of cell surface proteoglycans and glycoproteins, which have roles in tumor invasion, metastasis and resistance to chemotherapy and radiotherapy (9,21-23). A previous study that investigated nasopharyngeal carcinoma demonstrated an increased expression of CD44-positive cells that may have initiated increased rates of tumorigenesis and metastasis (11). In accordance with these findings, the present study revealed that the cancer stem-like SP cells exhibited an increased level of CD44 expression compared with the non-SP cells. Alongside ABCG2, CD44 may also contribute to chemotherapy resistance, tumorigenesis and the invasion of OS-55 cells. The data from the present study demonstrated the existence of cancer stem-like cells in the human OS-55 cell line. These cells possess stem cell features and high levels of self-renewal, and therefore, may be involved in tumor recurrence and metastasis. However, the functional interaction between the stem cell surface proteins, anti-apoptotic factors and $\mathrm{ABC}$ transporter proteins requires further investigation.

\section{References}

1. Marina N, Gebhardt M, Teot L and Gorlick R: Biology and therapeutic advances for pediatric osteosarcoma. Oncologist 9: 422-441, 2004

2. Damron TA, Ward WG and Stewart A: Osteosarcoma, chondrosarcoma, and Ewing's sarcoma: National Cancer Database report. Clin Orthop Relat Res 459: 40-47, 2007.

3. Jordan CT, Guzman ML and Noble M: Cancer stem cells. N Engl J Med 355: 1253-1261, 2006.

4. Lapidot T, Sirard C, Vormoor J, Murdoch B, et al: A cell initiating human acute myeloid leukaemia after transplantation into SCID mice. Nature 367: 645-648, 1994.

5. Hemmati HD, Nakano I, Lazareff JA, Masterman-Smith M, et al: Cancerous stem cells can arise from pediatric brain tumors, Proc Natl Acad Sci USA 100: 15178-15183, 2003.

6. Al-Hajj M, Wicha MS, Benito-Hernandez A, Morrison SJ and Clarke MF: Prospective identification of tumorigenic breast cancer cells. Proc Natl Acad Sci USA 100: 3983-3988, 2003.

7. Li C, Heidt DG, Dalerba P, et al: Identification of pancreatic cancer stem cells. Cancer Res 67: 1030-1037, 2007.

8. Kondo T, Setoguchi T and Taga T: Persistence of a small subpopulation of cancer stem-like cells in the C6 glioma cell line. Proc Natl Acad Sci USA 101: 781-786, 2004.

9. Wu C, Wei Q, Utomo V, Nadesan P, et al: Side population cells isolated from mesenchymal neoplasms have tumor initiating potential. Cancer Res 67: 8216-8222, 2007.

10. Gibbs CP, Kukekov VG, Reith JD, et al: Stem-like cells in bone sarcomas: implications for tumorigenesis. Neoplasia 7: 967-976, 2005.

11. Su J, Xu XH, Huang Q, et al: Identification of cancer stem-like CD44+cells in human nasopharyngeal carcinoma cell line. Arch Med Res 42: 15-21, 2011.

12. Goodell MA, Brose K, Paradis G, et al: Isolation and functional properties of murine hematopoietic stem cells that are replicating in vivo. J Exp Med 183: 1797-1806, 1996.

13. Pardal R, Clarke MF and Morrison SJ: Applying the principles of stem-cell biology to cancer. Nat Rev Cancer 3: 895-902, 2003.

14. Chang CC: Recent translational research: stem cells as the roots of breast cancer. Breast Cancer Res 8: 103, 2006.

15. Wang L, Park P and Lin CY: Characterization of stem cell attributes in human osteosarcoma cell lines. Cancer Biol Ther 8: 543-552, 2009.

16. Lee J, Kim HK, Rho JY, Han YM and Kim J: The human OCT-4 isoforms differ in their ability to confer self-renewal. J Biol Chem 281: 33554-33565, 2006.

17. Okita K, Ichisaka T and Yamanaka S: Generation of germline-competent induced pluripotent stem cells. Nature 448: 313-317, 2007.

18. Ezeh UI, Turek PJ, Reijo RA and Clark AT: Human embryonic stem cell genes OCT4, NANOG, STELLAR, and GDF3 are expressed in both seminoma and breast carcinoma. Cancer 104: 2255-2265, 2005.

19. Looijenga LH, Stoop H, de Leeuw HP, et al: POU5F1 (OCT3/4) identifies cells with pluripotent potential in human germ cell tumors. Cancer Res 63: 2244-2250, 2003.

20. Cheng L: Establishing a germ cell origin for metastatic tumors using OCT4 immunohistochemistry. Cancer 101: 2006-2010, 2004.

21. Setoguchi T, Taga T and Kondo T: Cancer stem cells persist in many cancer cell lines. Cell Cycle 3: 414-415, 2004.

22. Pesce M and Schöler HR: Oct-4: gatekeeper in the beginnings of mammalian development. Stem Cells 19: 271-278, 2001.

23. Rodda DJ, Chew JL, Lim LH, et al: Transcriptional regulation of nanog by OCT4 and SOX2. J Biol Chem 280: 24731-24737, 2005. 